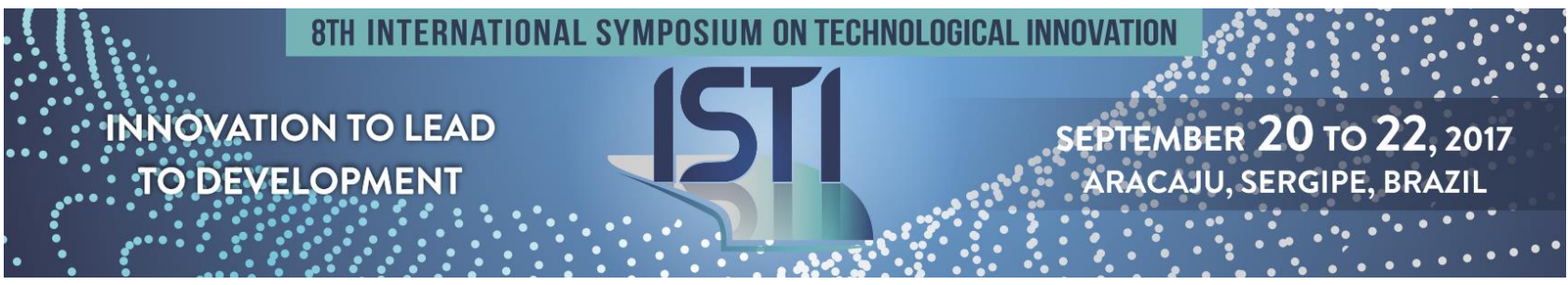

\title{
LEVANTAMENTO BIBLIOGRÁFICO SOBRE A PROPRIEDADE INTELECTUAL A PARTIR DA LEI DE INOVAÇÃO
}

\author{
Jandira Reis Vasconcelos - jandirasantosreis@yahoo.com.br \\ Discente do Programa de Pós-Graduação em Ciência da Propriedade Intelectual - UFS \\ João Antonio Belmino dos Santos - joaoantonio@ufs.br \\ Doutor em Engenharia de Processos- UFCG \\ Docente do Programa de Pós-Graduação em Ciência da Propriedade Intelectual - UFS
}

Resumo- A Propriedade Intelectual (PI) tem demonstrado interesse em diversas áreas do conhecimento, pois diz respeito à criação da mente humana. Com a criação Lei de Inovação de 2004, a PI inseriu-se em um novo contexto ao contar com dispositivo legal para o incentivo a inovação e a pesquisa científica e tecnológica. Com o objetivo de verificar o panorama de pesquisas sobre Propriedade Intelectual de 2004 a 2017, foi feito um levantamento bibliográfico nas bases de dados Scopus e Web of Science, onde foram extraídos respectivamente 8.834 e 5.780 artigos científicos. Foi mensurado o quantitativo de artigos publicados por ano, por área do conhecimento e por países. A análise dos dados permitiu destacar que em ambas as bases houve oscilação de publicações sobre o tema nos anos pesquisados, os Estados Unidos lideram com maior número de pesquisas, o Brasil está inserido, mesmo apresentando um número ainda incipiente, diversas áreas do conhecimento demonstraram interesse, mas as Ciências Sociais teve destaque na Scopus e a Economia de Negócios na Web of Science.

Palavras-chave: Propriedade Intelectual, Produção científica, Inovação, Base de dados.

Abstract - The Intellectual Property (IP), has shown interest in several areas of knowledge, as it concerns the creation of the human mind. With the creation of the Innovation Law of 2004, PI inserted itself in a new context by having a legal device to encourage innovation and scientific and technological research. In order to verify the panorama of researches on Intellectual Property from 2004 to 2017, a bibliographic survey was made in the Scopus and Web of Science databases, where 8,834 and 5,780 scientific articles were extracted respectively. We measured the number of articles published per year by area of knowledge and by country. The analysis of the data allowed to highlight that in both bases there was oscillation of publications on the subject in the years surveyed, the United States leads with more research, Brazil is inserted, even presenting a still incipient number, several areas of knowledge have shown interest, But Social Sciences excelled in Scopus and Business Economics in the Web of Science.

Keywords- Intellectual Property, Scientific production, Innovation, Database. 


\section{INTRODUÇÃO}

A Propriedade Intelectual, de acordo com a Organização Mundial da Propriedade Intelectual (OMPI), diz respeito a toda criação da mente humana, seja no campo científico, tecnológico, artístico ou literário. Segundo Ferraz e Basso (2011) está dividida em três categorias: a Propriedade Industrial, o Direito Autoral e a Proteção Sui Generis. Relacionam-se com várias áreas do conhecimento, por abranger temas diversificados, como patente, marca, modelo de utilidade, desenho industrial, indicação geográfica, cultivar e programa de computador, entre outras.

De acordo com a explanação, percebe-se o quanto a Propriedade Intelectual é abrangente, assumindo uma característica interdisciplinar. Neste sentido, Borher et al. (2007) ao discutirem a necessidade de organização de iniciativas em torno de educação e pesquisa em Propriedade Intelectual, destacam evidência de perspectiva interdisciplinar para análise do tema propriedade intelectual no meio acadêmico.

Como advento da Lei de Inovação de 2004, a Propriedade Intelectual passa a contar com um dispositivo legal que promove à inovação no campo da ciência e da tecnologia (PAIXÃO, 2016). Dessa forma acaba por favorecer o desenvolvimento das pesquisas científicas nas Universidades. Neste sentido, os pesquisadores contam como veículo de divulgação das pesquisas, os periódicos científicos, que por sua vez são indexados em bases de dados. No que diz respeito à Propriedade Intelectual no contexto do periódico científico, Mueller (2003) afirma que o período assume também a função de Propriedade Intelectual, pois autor ao divulgar suas pesquisas, torna formal sua autoria e adquire a prioridade na descoberta científica.

Dentro desse contexto, este artigo tem por objetivo verificar o quantitativo das pesquisas sobre o tema Propriedade Intelectual, em periódicos científicos, indexados nas bases de dados Scopus e Web of Science. Especificamente objetiva verificar as publicações por ano, por área do conhecimento e por países.

As informações e reflexões que foram levantadas e produzidas estão apresentadas nas seções subseqüentes a esta introdução, sendo o referencial teórico, a metodologia, a análise e discussão dos resultados e finalizando com as considerações finais.

\section{REFERENCIAL TEÓRICO}

No referencial teórico estão apresentados alguns tópicos de relevância para o contexto do tema, como forma de embasamento ao desenvolvimento desse estudo. Dessa forma, foram abordadas algumas teorias referentes à produção científica, inovação, bem como sobre as bases de dados Scopus e Web of Science.

\section{1. PRODUÇÃO CÍENTÍFICA}

A produção científica é o resultado de pesquisas publicadas em meios formais ou informais, que podem ser registradas em dissertações, teses, artigos de periódico, comunicações apresentadas em congressos, monografias, folhetos e capítulos de livros. Esses meios de informações servem de base para os estudos bibliométricos, além de colaborarem para a história social do conhecimento (ARAÚJO e ALVARENGA, 2011). De acordo com os autores, é válido salientar, que a produção intelectual do conhecimento científico, pode ser avaliada utilizando como suporte os métodos da bibliometria. 
Sacardos e Hayashi (2013) corroboram com este argumento, ao considerar a bibliometria, como um conjunto de métodos que valem de ferramentas e modelos matemáticos e estatísticos para análises quantitativas e comparativas da produção científica. Diversas áreas do conhecimento têm adotado tal metodologia para avaliar o potencial quantitativo das pesquisas. Neste sentido, Almeida, Oliveira e Russo (2016) afirmam que a bibliometria se constitui como uma ferramenta com potencialidade para análise da produção científica, crucial ao pesquisador, instituições e grupos de pesquisas.

\subsection{INOVAÇÃO}

A inovação, segundo o Manual de OSLO (2005) diz respeito a realização de um bem ou serviço, novo ou melhorado, podendo ser: um processo, um método nas práticas de negócios, no ambiente de trabalho ou relações externas.

De acordo com a Lei de Inovação de 2004, que dispõe sobre incentivos à inovação e à pesquisa científica e tecnológica no ambiente produtivo, a inovação pode ser entendida como "introdução de novidade ou aperfeiçoamento no ambiente produtivo ou social que resulte em novos produtos, processos ou serviços". Assim as pesquisas científicas e as inovações ganham forças no meio acadêmico, com a criação dos Núcleos de Inovação Tecnológicas, previsto na Lei, que tem como objetivo o desenvolvimento da política de inovação (Borges, 2015).

Nesse sentido, os resultados das inovações, nas universidades, através das pesquisas científicas, são publicados em periódicos científicos, para que a sociedade tenha mais possibilidades de acesso ao conhecimento produzido no meio acadêmico, de forma precisa e confiável.

\subsection{BASES DE DADOS SCOPUS E WEB OF SCIENCE}

As bases de dados constituem-se como importantes ferramentas de buscas para pesquisas, são abrangentes, reúnem informações estruturadas e permitem encontrar resultados da produção científica de determinada área do conhecimento, bem como instrumentos que dão suportes à revisão bibliográfica e à levantamentos bibliométricos. (OLIVEIRA, GRÁCIO, 2011 ; SILVA, 2013).

A Scopus, desenvolvida pela editora Elsevier, é a maior base de dados interdisciplinar de referências bibliográficas e resumo de literatura revisada por pares, com abrangência mundial, oferece pesquisa nas áreas de ciência, tecnologia, medicina, ciências sociais, artes e humanidades (ELSEVIER, 2017).

A Web of Science (WoS), junto a Thomson Reuters Scientific, oferece acesso a referências e resumos em todas as áreas do conhecimento. Disponibiliza ferramentas que possibilitam análise de citações, referências, índice $\mathrm{h}$, permitindo análises bibliométricas. Cobre aproximadamente 12.000 periódicos. Disponibiliza consulta a 5 coleções que contêm informações multidisciplinares: Science Citation Index Expanded (SCI-EXPANDED); Social Sciences Citation Index (SSCI); Arts\&Humanities Citation Index (A\&HCI); Conference Proceedings Citation Index-Science (CPCI$S)$ e Conference Proceedings Citation Index - Social Science \&Humanities (CPCI-SSH). (CAPES; MEC 2017). 


\section{METODOLOGIA}

Desenvolveu-se uma pesquisa bibliométrica, com abordagens quantitativas, em artigos de periódicos científicos, relacionados ao tema Propriedade Intelectual. A definição da amostra teve como marco temporal, o período de 2004 a 2017.

A coleta de dados foi feita através do Portal de Periódicos da Capes, onde se acessou as bases de dados Scopus e Web of Science. Os dados foram coletados no início do mês de julho de 2017.A escolha às essas fontes se deu pelo fato de as mesmas disponibilizarem informações científicas, atualizadas, com abrangência nacional e por conterem artigos publicados em múltiplas áreas do conhecimento.

A estratégia de busca foi o uso do termo "Intellectual property", na pesquisa avançada, nos campos: título, resumo e palavras-chave. Em seguida foi feito o refinamento para a escolha do tipo de documento, selecionando a opção artigo de periódico. Em seguida feito o levantamento dos dados, de forma a atender os objetivos específicos, abordando o quantitativo de artigos publicados por ano, por área do conhecimento e por países.

Quanto aos procedimentos de análise dos dados efetuou-se um exame das informações coletadas a partir das bases de dados, realizando um tratamento, através do Software Excel e a partir do qual se obteve os resultados que alimentaram a construção dos gráficos.

\section{RESULTADOS E DISCUSSÃO}

A busca pelo termo "Intellectual property" na base de dados Scopus, nos campos " Articletitle, Abstract, Keywords", permitiu encontrar 8.875 artigos de periódicos publicados. As figuras 1,2 e 3 representam o cenário quantitativo dos dados, por ano, bem como por área do conhecimento e por países.

Figura 1. Publicações de 2004 a 2017

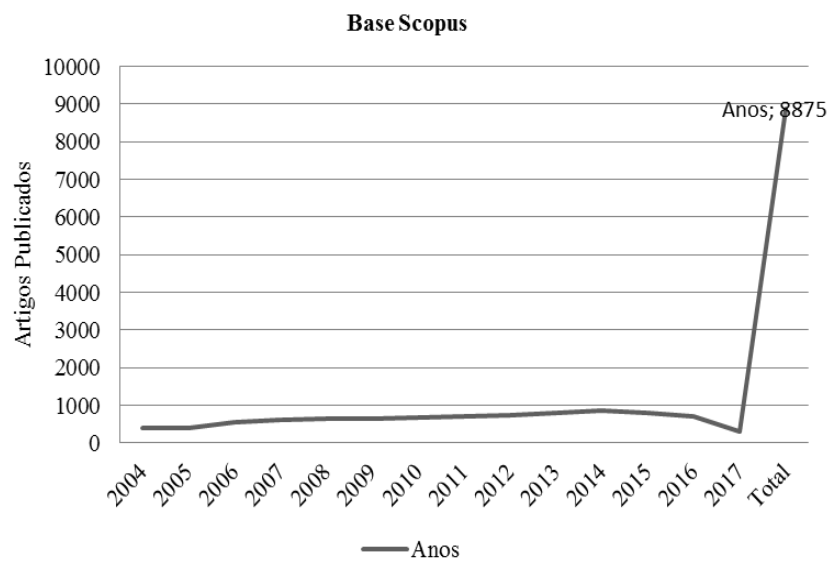

Fonte: Elaborado pelos autores a partir de Scopus (2017)

A Figura 1 apresenta o quantitativo, das publicações de artigos de periódicos, indexados na base de dados Scopus, no período de 2004 a 2017, atingindo 8.875 publicações. Pode-se observar que no recorte temporal houve ascensão das publicações a partir de 2004, que contava com 402 artigos. O 
auge das publicações se deu no ano de 2014, com um quantitativo de 861, porém a partir de 2015 houve um declínio do número de trabalhos publicados.

Figura 2. Publicações por área do conhecimento

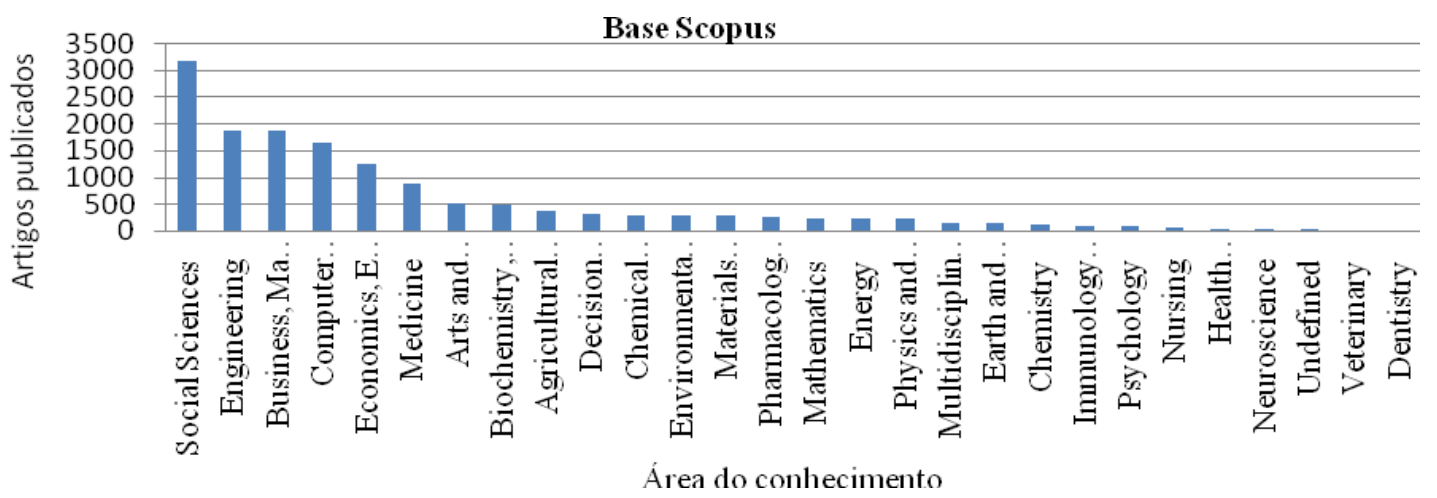

Fonte: Elaborado pelos autores a partir de Scopus (2017)

A Figura 2 mostra as publicações por área do conhecimento. Percebe-se que a Propriedade Intelectual desperta interesse de pesquisa em múltiplas áreas, porém a que mais se destaca são as Ciências Sociais, com 3221 artigos, seguida de Engenharia com 1888, Ciência da Computação 1655, Ciências Econômicas 1269, Medicina 879. A agricultura apresenta o menor número de pesquisas nesta temática.

Figura 3. Publicações por países

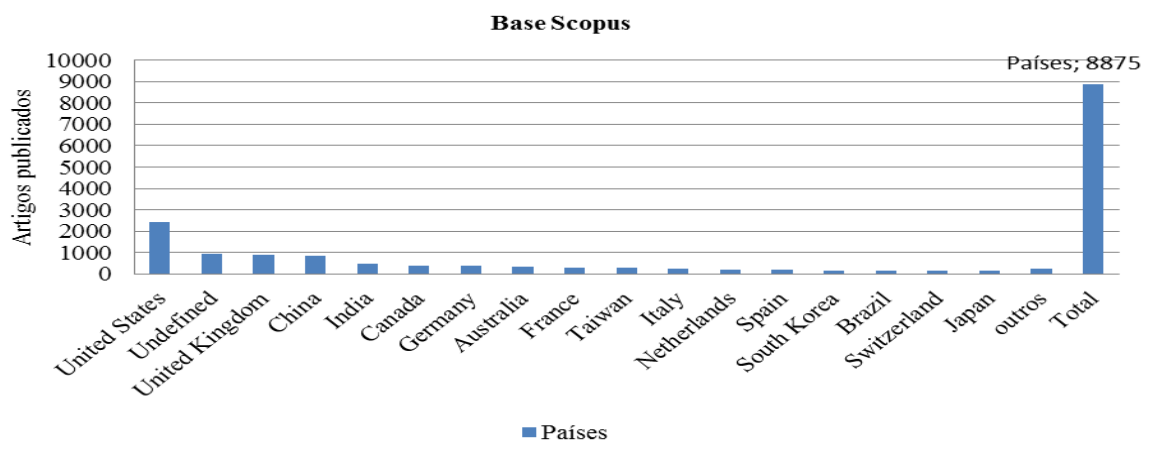

Fonte: Elaborado pelos autores a partir de Scopus (2017)

A Figura 3 apresenta o panorama com 17 países que mais publicaram. Observa-se que o os Estados Unidos lideram com o maior número de publicações, apresentando 2427 artigos, à frente do Reino Unido, China, Índia, Canadá, Alemanha, Austrália, França, Taiwan entre outros.

O Brasil, apesar de apresentar apenas 165 publicações, ainda assim destaca-se sobre a Suíça e o Japão por possuírem menor número de pesquisas na área em comparação aos demais países. 
Com relação à pesquisa na Web of Science, utilizando a mesma estratégia de busca, foram encontrados 5.780 artigos de periódicos indexados. As figuras 4, 5 e 6, ilustram o quantitativo dessa produção científica distribuídos por ano, área do conhecimento e países.

Figura 4. Publicações de 2004 a 2017

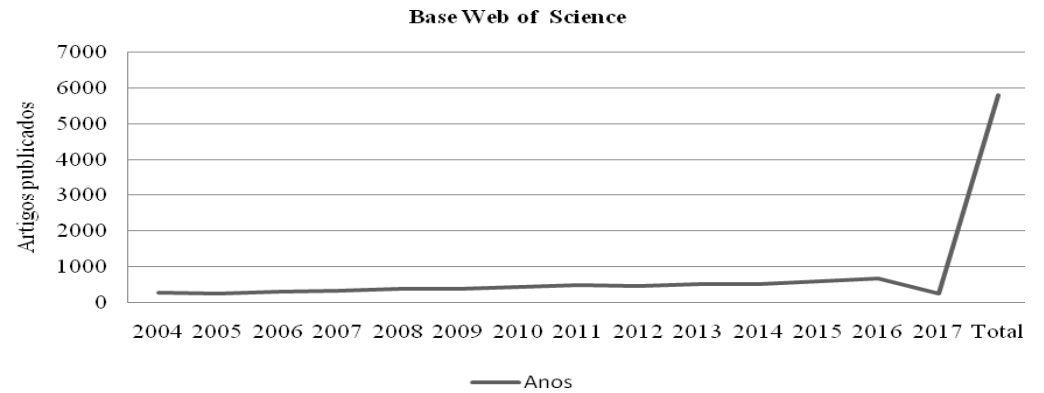

Fonte: Elaborado pelos autores a partir de Web of Science (2017)

Os dados da Figura 4 expressam o panorama do quantitativo por ano de 2004 a 2017, totalizando 5.780 artigos. Percebe-se que houve oscilação das produções científicas, entre os anos de 2004 a 2015, porém o ano de 2016 teve o maior pico, atingindo 665 publicações. Em 2017 encontrase o menor número, apenas 254, justificável por estar em curso, porém apresenta possibilidade de evolução desse número se comparar ao ano de 2004, que apresentou 270 publicações.

Figura 5. Publicações por área do conhecimento

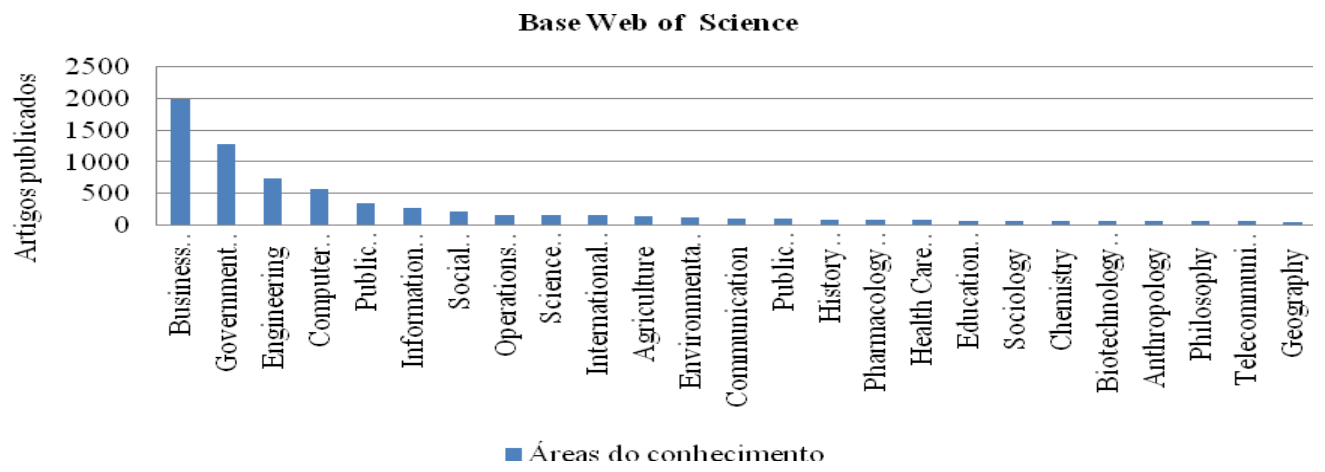

Fonte: Elaborado pelos autores a partir de Web of Science (2017)

Apresenta-se na Figura 5, o quantitativo por área do conhecimento, percebe-se que, vários campos do saber desenvolvem pesquisas sobre a Propriedade Intelectual, porém a Economia de Negócios teve destaque com 1996 publicações. Ressaltam-se também outras áreas de interesse, como a Engenharia, Ciência da Computação, Administração Pública, Ciência da Informação, Ciências 
Sociais, e demais elencadas na figura. A geografia apresenta o menor número de publicações, apenas 56 artigos.

Figura 6. Publicações por países

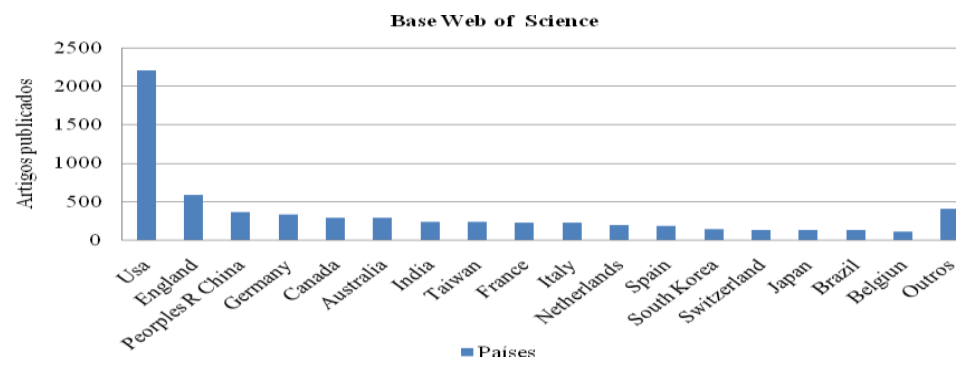

Fonte: Elaborado pelos autores a partir de Web of Science (2017)

Na Figura 6, estão apresentados os países com maior número de publicações sobre o tema. Os Estados Unidos ocupam o primeiro lugar, com 2203 publicações, à frente da Inglaterra, China, Alemanha, Canadá e demais países apresentados. O Brasil ocupa o $16^{\circ}$ lugar com apenas 123 publicações, apesar desta colocação, está à frente da Bélgica e outros países.

\section{CONSIDERAÇÕES FINAIS}

Verifica-se uma vasta produção científica sobre a Propriedade Intelectual, distribuída em vários países do mundo, bem como em áreas distintas do conhecimento. Isso reflete a relevância desse tema para as pesquisas no meio científico.

Em uma análise comparativa, percebe-se que a base Scopus possui um maior número de artigos indexados, por conter 8875 publicações, já a na Web of Science foram encontrados apenas 5812 artigos publicados. No que diz respeito à análise temporal, em ambas as bases houve variação no quantitativo das pesquisas nos anos abordados, porém apesar da variação, pode-se constatar que há uma tendência de crescimento do número de publicações.

$\mathrm{Na}$ avaliação por área do conhecimento, foi possível perceber que a Propriedade Intelectual desperta interesse por várias áreas nas bases pesquisadas. Os destaques estão para as Ciências Sociais e Economia de Negócios, as áreas com menor produção são Agricultura e Geografia, respectivamente na Scopus e Web of Science.

Quanto à contribuição por países, os Estados Unidos apresentam-se em primeiro lugar nas duas bases. No Brasil não há uma grande produção científica sobre o tema abordado, o número ainda é incipiente, apesar de estar à frente de outros países.

\section{REFERENCIAS}

ALMEIDA, Glessiane Oliveira; OLIVEIRA, Luana Brito de; RUSSO, Suzana Leitão. Noções de bibliometria: um passo a passo. In: RUSSO, Suzana Leitão et al. (orgs.). Propriedade intelectual, tecnologias e empreendedorismo. Aracaju: API, 2016. 
ARAUJO, Ronaldo Ferreira; ALVARENGA, Lidia. A bibliometria na pesquisa científica da pósgraduação brasileira de 1987 a 2007. Revista Eletrônica de Biblioteconomia e Ciência da Informação, Florianópolis, v. 16, n. 31, p.51-70, 2011. Disponível em: <periodicos.ufsc.br>. Acesso em: 08 dez. 2016.

BARCELOS, Régis Leonardo Gusmão; MOCELIN, Daniel Gustavo. Ciência e mercado: impasses na institucionalização de práticas empreendedoras em uma universidade pública brasileira. Revista Brasileira de Ciências Sociais. v. 31, n. 92 out./2016. Disponível em: 〈http://www.scielo.br>. Acesso em: 27 jun. 2017.

BORGES, Maria Creusa de Araújo. Regulação da educação superior brasileira: a Lei de Inovação Tecnológica e da Parceria Público-Privada. Educação e Pesquisa. São Paulo, v. 41, n. 4, p. 961-973, out./dez. 2015. Disponível em:< http://www.scielo.br>. Acesso em: 28 jun. 2017.

BORHER, Maria Beatriz Amorim et al. Ensino e pesquisa em propriedade intelectual no Brasil. Revista Brasileira de Inovação, Rio de Janeiro (RJ), 6 (2), p.281-310, jul/dez 2007. Disponível em: <https://ocs.ige.unicamp.br/ojs/rbi/article/view/321/240>. Acesso em: 19jun. 2017.

BRASIL. Lei $\mathrm{n}^{\mathrm{o}} 10.973$, de 02 de dezembro de 2004. Disponível em:<http://www.planalto.gov.br/ccivil_03/_ato2004-2006/2004/lei/110.973.htm>. Acesso em: 9 jun. 2017.

CAPES; MEC.Web of Science: coleção principal. Portal de periódicos CAPES/MEC. Disponível em: <http://www-periodicos-capes-gov-br.ez20.periodicos.capes.gov.br>. Acesso em: 21 jun. 2017.

ELSEVIER. Scopus. Disponível em: <https://www.elsevier.com/americalatina/pt-br/scopus>. Acesso em: 12 jul. 2017.

MANUAL DE OSLO: diretrizes para coleta e interpretação de dados sobre inovação. 3. ed., OECD: FINEP, 2005. Disponível em: <http://www.finep.gov.br>. Acesso em: 19 jun. 2017.

MULLER, Suzana Pinheiro Machado. O periódico científico. In: CAMPELO, Bernadete Santos; CEDÓN, Beatriz Valadares; KREMER, Jeannette Marguerite (Orgs.). Fontes de informação para pesquisadores e profissionais. Belo Horizonte: Ed. UFMG, 2003.

PAIXÃO, Ana Eleonora Almeida. Legislação e contextualização. In: RUSSO, Suzana Leitão ...et al. (orgs.). Propriedade intelectual : um guia em forma de questões. Aracaju : Associação de Propriedade Intelectual, 2016.

SACARDO, Michele Silva; HAYASHI, Maria Cristina Piumbato Innocentini. Bibliometria e epistemologia: balanços iniciais da produção científica em educação física na interface com a educação. In:

HAYASHI, Maria Cristina PiumbatoInnocentini (Org.). Bibliometria e cientometria: estudos temáticos. São Carlos: Pedro \& João, 2013. 
SCOPUS (Elsevier). Disponível em: < https://www-scopuscom.ez20.periodicos.capes.gov.br/home.uri>.

WEB OF SCIENCE. Disponível <http://appswebofknowledge.ez20.periodicos.capes.gov.br/WOS_GeneralSearch_input.do?product=WOS\&searc h_mode=GeneralSearch\&SID=2EGHr9pip8gy6kmP533\&preferencesSaved $>$. 